\title{
Cumulative radiation dose incurred during the management of complex pleural space infection
}

\author{
Christopher R. Gilbert ${ }^{*}$, Anee S. Jackson², Candice L. Wilshire', Leah C. Horslen', Shu-Ching Chang 3 , \\ Adam J. Bograd ${ }^{1}$, Eric Vallieres ${ }^{1}$ and Jed A. Gorden ${ }^{1}$
}

\begin{abstract}
Background: Complex pleural space infections are commonly managed with antibiotics, pleural drainage, intrapleural fibrinolytic therapy, and surgery. These strategies often utilize radiographic imaging during management, however little data is available on cumulative radiation exposure received during inpatient management. We aimed to identify the type and quantity of radiographic studies along with the resultant radiation exposure during the management of complex pleural space infections.
\end{abstract}

Methods: Retrospective review of community network healthcare system from January 2015 to July 2018. Patients were identified through billing databases as receiving intrapleural fibrinolytic therapy and/or surgical intervention. Patient demographics, clinical outcomes, and inpatient radiographic imaging was collected to calculate cumulative effective dose.

Results: A total of 566 patients were identified with 7275 total radiographic studies performed and a median cumulative effective dose of 16.9 (IQR 9.9-26.3) mSv. Multivariable linear regression analysis revealed computed tomography use was associated with increased cumulative dose, whereas increased age was associated with lower cumulative dose. Over $74 \%$ of patients received more than $10 \mathrm{mSv}$, with $7.4 \%$ receiving more than $40 \mathrm{mSv}$.

Conclusions: The number of radiographic studies and overall cumulative effective dose in patients hospitalized for complex pleural space infection was high with the median cumulative effective dose $>5$ times normal yearly exposure. lonizing radiation and modern radiology techniques have revolutionized medical care, but are likely not without risk. Additional study is warranted to identify the frequency and imaging type needed during complex pleural space infection management, attempting to keep ionizing radiation exposure as low as reasonably possible.

Keywords: Complex pleural space infection, Cumulative effective dose, Radiation exposure, Radiation safety, Empyema management

\footnotetext{
*Correspondence: christopher.gilbert@swedish.org

${ }^{1}$ Thoracic Surgery and Interventional Pulmonology, Swedish Cancer

Institute, 1101 Madison St, Suite 900, Seattle, WA 98104, USA

Full list of author information is available at the end of the article
}

\begin{abstract}
Introduction
Parapneumonic effusions are common and are often treated with antibiotics and/or pleural drainage. Although less common, more complicated pleural disease in the setting of pneumonia, such as complicated parapneumonic effusions and empyema can carry a high morbidity and mortality and often require additional interventions. The current management of complex
\end{abstract}


pleural space infections (CPSI) generally requires antibiotics, pleural space drainage, and in some cases will require additional interventions such as intrapleural fibrinolytic therapy (IPFT) and/or surgery (evacuation and/or decortication). Published guidelines exist on the management of infectious pleural disease itself [1,2], however little attention has been paid to radiation exposure that may be incurred during the management of these processes and interventions.

Diagnostic and therapeutic imaging of the chest often includes single (portable anteroposterior) or two (posteroanterior and lateral) view chest $\mathrm{x}$-ray, computed tomography of the chest, and fluoroscopy-all of which produce ionizing radiation to the patient and sometimes healthcare staff. These imaging modalities are generally considered "routine" and utilized during the initial evaluation as well as during ongoing management of pneumonia and CPSI. We are unaware of any recommendations or attempts at standardization/minimization of radiation exposure during CPSI management. Globally speaking, the medical field has moved towards minimizing radiation exposure to patients as reasonable in order to provide adequate care, as most believe there is increased risk of carcinoma with increased exposure to ionizing radiation [3]. As a result of these concerns, attention has been called to radiation during medical care in certain populations, including trauma patients $[4,5]$, children [6-8], ICU care [9, 10], and general hospital care [11]. However, lack of awareness and guidelines related to radiation exposure during CPSI management has the potential for significant practice variation and subsequent differences in radiographic study use and radiation exposure. We aimed to determine the number and type of radiographic studies performed and the resultant radiation cumulative effective dose during the management of CPSI within a large, multicenter healthcare network.

\section{Methods}

\section{Data sources and study population}

A retrospective review of patients undergoing management of complicated pleural space infection from January 2015 to July 2018 within a multistate community-based network of 18 hospitals was performed. The SwedishProvidence Health Care system is a community-based network located in the Western United States, covering Alaska, Washington, Idaho, Montana, Oregon, and California. An ethics committee, The Institutional Review Board at Swedish Medical Center approved this study, and a waiver of consent was granted (Study\# 2018000200). All methods were performed in accordance with relevant guidelines, practices, and regulations related to performing human subject research at Swedish Medical Center.
All patients were initially identified from a central inpatient billing database. IPFT use was identified from pharmacy billing records of intrapleural dornase. Surgical intervention was identified from procedural codes for thoracotomy or thoracoscopy with or without decortication in the setting of chest tube insertion and diagnosis of pleural infection/empyema (Table 1).

Crossover therapy was identified when both modalities were utilized within the same hospitalization (i.e., IPFT followed by surgery). Inclusion criteria included receiving IPFT or surgical intervention for management of a CPSI. Exclusion criteria included known malignant/paramalignant pleural effusion, hemothorax, empyema related to esophageal perforation, incomplete medical record (i.e., receiving initial care at an outside hospital network), current in situ indwelling tunneled pleural catheter, and/ or prior chest surgery. Patient characteristics including demographics, management strategy for CPSI, chest tube duration, and hospital stay were collected. Chart data was also reviewed to formulate a RAPID score $[12,13]$ at the time of chest tube placement. Review of the electronic medical record of relevant imaging reports was performed.

\section{Radiation dose estimates}

All radiographic studies from the initial date of CPSI identification to completion of management were captured. A brief review of relevant medical physics and the rationale for using cumulative effective dose (CED) can be identified in previous papers, including one by Kim et al. [4] identifying CED in trauma patients. As a brief

Table 1 CPT and ICD billing codes associated with complex pleural space infection and related interventions

\begin{tabular}{lll}
\hline CPT code & ICD-9 code & $\begin{array}{l}\text { ICD-10 } \\
\text { code }\end{array}$ \\
\hline 32652 & 34.52 & OBDN4ZZ \\
32651 & 34.51 & OBDNOZZ \\
32220 & 34.24 & OBDP4ZZ \\
32225 & 34.59 & OBDNOZX \\
32320 & 510.0 & OBDN3ZZ \\
32653 & 510.9 & OBDN4ZX \\
32551 & 511.0 & OBDPOZZ \\
32556 & 511.1 & OBDP3ZX \\
32557 & 511.9 & OBDP4ZX \\
& & OBBPOZX \\
& & OBBPOZZ \\
& & OBBNOZX \\
& & OBBNOZZ \\
& & J86.0 \\
& & J86.9 \\
& & ROO.1 \\
& & J90 \\
& &
\end{tabular}


synopsis, the standard value for radiation dose estimate (and often reported in medical journals) collected is the sievert $(\mathrm{Sv})$ or millisievert $(\mathrm{mSv})$. Other reports may describe radiation exposure units as gray (Gy), however both Sv and Gy are expressed in Joule/Kilogram and often utilized when discussing radiation and exposuresall leading to confusion, [14] as they are different. At a very basic level, the General Conference of Weights and Measures has decided that Gy be used for the absorbed dose (commonly utilized for radiotherapy dosing) and Sv be used for the dose equivalent (commonly used in radiation protection). The exposure of radiation to human tissue and its effects (cancer, etc.) depends on the magnitude of the dose equivalent/estimate [14], and hence use of $\mathrm{Sv}$ and $\mathrm{mSv}$ in many human radiation exposure papers.

References of the dose estimates for each type of imaging study was obtained from previously published references $[11,15]$. Chest fluoroscopy and CT guidance for placement of chest tubes are not routinely identified with a reference radiation dose in $\mathrm{mSV}$. They are time dependent procedures and the dose equivalent can vary depending on the trajectory and size of the radiation beam. Therefore, we elected to average the dose equivalent based on dose length product (DLP) and dose area product (DAP) [16] for these procedures. The CED of a patient was calculated as the sum of all effective doses.

\section{Statistical analysis}

All data was collected and stored in Excel (Microsoft, Redmond, WA). Simple descriptive statistics were used to report demographics and outcomes. Univariate linear regression analyses were first used to identify factors associated with total CED, followed by multivariate linear regression analyses. Due to concerns related to collinearity associated with total CED and variables directly related to radiation (i.e., CT scan) we elected to remove this variable from the multivariate analysis. However did want to test the impact of image guided tube thoracostomy (CT and fluoroscopic guidance) understanding there was also likely some collinearity associated with these variables, therefore we elected to run two multivariate models. Statistical significance was defined as $p<0.05$. All statistical analyses were performed using SPSS 24.0 statistical software package (SPSS Inc., Chicago, IL, USA) and R version 3.6.0 (R Core Team 2019).

\section{Results}

A total of 1,640 patients were identified as meeting inclusion criteria from billing records. After chart review patients were excluded for the following reasons: malignant/paramalignant pleural effusion-201, hemothorax-186, empyema related to esophageal perforation-89, incomplete medical record-117, current in situ indwelling tunneled pleural catheter-25, and prior chest surgery-456. A total of 566 patients were therefore eligible and included in the analysis.

Patient demographics and hospital stay information is available in Table 2.

The median age of our population was 58 (IQR 46-68) years, composed of mostly males $(66 \%-374 / 566)$ and the majority of infections were community acquired 88\% (498/566). The median RAPID score was 3 (IQR 2-4). The median overall length of stay (LOS) was 11 (IQR 8-16) days and median duration of chest tube drainage was 6 (IQR 4-9) days. The majority of patients (85\%) were managed in hospitals with thoracic surgeon presence.

The overall number of radiographic studies performed was 7275 , with the majority of imaging, $(60.2 \%)$ being a single view chest radiograph. However, the majority of radiation exposure was related to diagnostic CT imaging. Exposure to diagnostic CT imaging (combining both CT angiogram and CT chest), accounted for $92 \%$ of the total CED within our population (Fig. 1). A total of $2.5 \%$ $(14 / 566)$ of the population did not receive a CT scan during their admission, whereas those undergoing CT imaging, $67.5 \%(382 / 566)$ underwent two or more CT scans.

The median CED for the entire population was 16.9 (IQR 9.9-26.3) $\mathrm{mSv}$, respectively, with a range of 0.1$73.4 \mathrm{mSv}$. Over $74 \%$ of patients received more than $10 \mathrm{mSv}$ during their hospital stay with $7.4 \%$ receiving more than $40 \mathrm{mSv}$ (Fig. 2).

Univariate linear regression analysis (Table 3 ) revealed that the following variables were significantly associated with a higher CED: increased hospital length of stay (continuous variable), hospital length of stay $>7$ days, total number of $\mathrm{CT}$ scans (continuous variable), having more than 1 CT performed, chest tube duration, evidence of abscess or necrotizing pneumonia on initial $\mathrm{CT}$, and use of CT or fluoroscopic guidance for chest tube placement.

Table 2 Patient demographics and hospital stay information

\begin{tabular}{ll}
\hline Demographic and hospital stay information & \\
\hline Age (median, IQR), years & $58(46-68)$ \\
Male/Female (n, \%) & $374(66 \%) / 192(44 \%)$ \\
Community Acquired Infection ( $n, \%)$ & $498(88 \%)$ \\
RAPID Score (median, IQR) & $3(2-4)$ \\
Body Mass Index (median, IQR) & $26.5(22.7-30.7)$ \\
Admitted to hospital with Thoracic Surgery (n, \%) & $479(85 \%)$ \\
Hospital length of stay (median, IQR) & $11(8-16)$ \\
Intensive care unit stay (median, IQR) & $1(0-3)$ \\
Duration of chest tube drainage (median, IQR) & $6(4-9)$ \\
Duration of IPFT instillation (median, IQR) & $3(3-4)$ \\
\hline
\end{tabular}




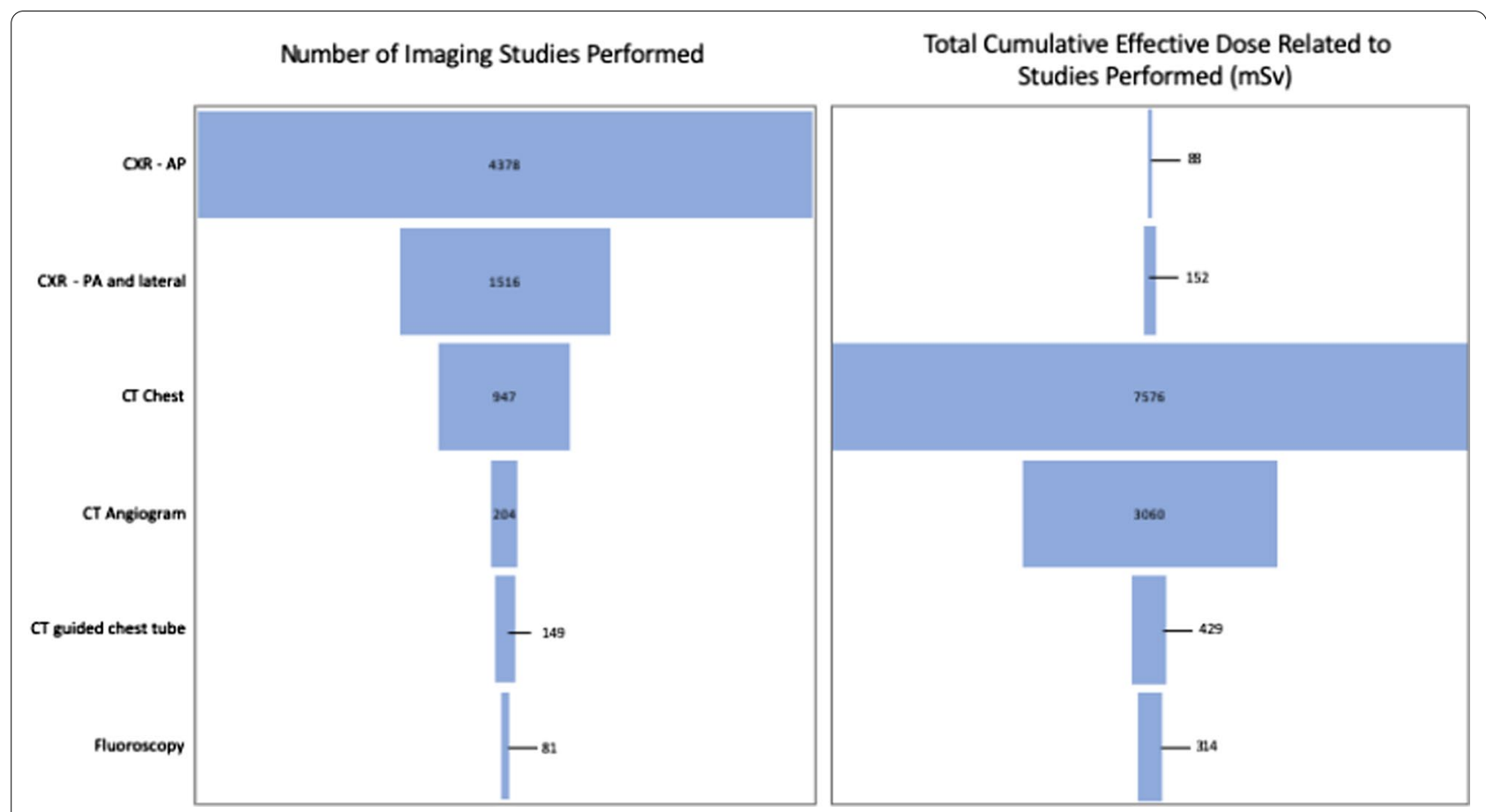

Fig. 1 a Graphical representation of the total number of imaging studies performed within the population, categorized by type of imaging. The type of imaging performed is listed along the vertical axis and the total number of each imaging type is displayed within the shaded bar. $\mathbf{b}$ Graphical representation of the total cumulative dose (in millisieverts ( $\mathrm{mSv}$ )) within the population, categorized by type of imaging. Again, the imaging modality is listed on the vertical axis and the total cumulative effective dose is listed within the shaded bar

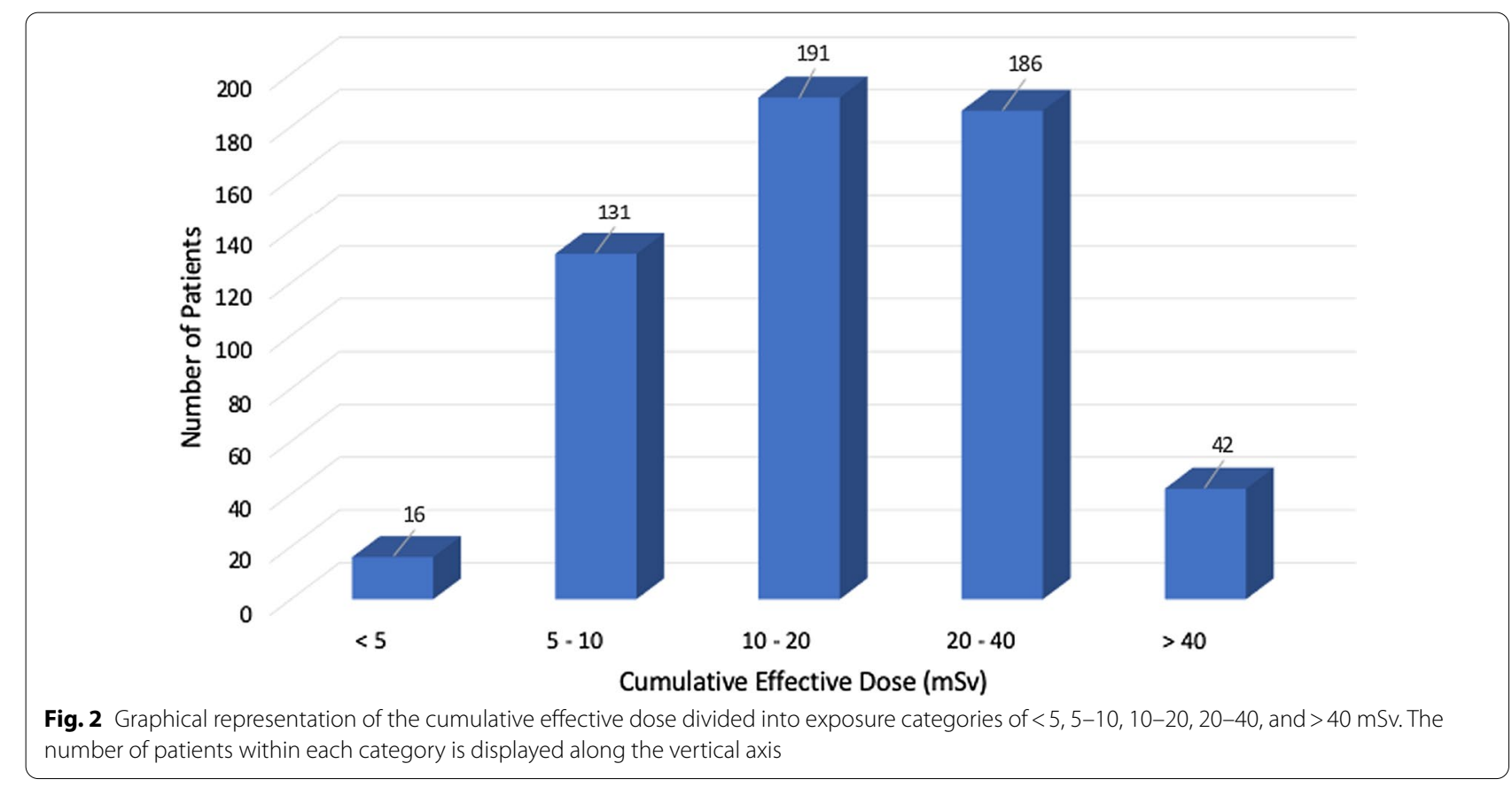


Table 3 Univariate and Multivariate linear regression analysis

\begin{tabular}{|c|c|c|c|c|}
\hline Variable & $\begin{array}{l}\text { Total CED mean } \\
\text { difference }\end{array}$ & Lower $95 \% \mathrm{Cl}$ & Upper $95 \% \mathrm{Cl}$ & $p$ value \\
\hline \multicolumn{5}{|l|}{ Univariate linear regression analysis } \\
\hline Hospital has thoracic surgery presence (Baseline: No) & -1.233 & -4.065 & 1.598 & 0.393 \\
\hline Surgical Treatment (Baseline: Medical) & -8.446 & -10.504 & -6.388 & $<0.001$ \\
\hline Crossover Treatment (Baseline: Medical) & 2.374 & -0.770 & 5.518 & 0.139 \\
\hline Body Mass Index & 0.027 & -0.114 & 0.169 & 0.705 \\
\hline Body Mass Index 20-<30 (Baseline: <20) & 1.781 & -1.999 & 5.561 & 0.355 \\
\hline Body Mass Index 30-<40 (Baseline: <20) & 1.462 & -2.743 & 5.666 & 0.495 \\
\hline Body Mass Index > 40 (Baseline: <20) & 0.713 & -4.605 & 6.031 & 0.792 \\
\hline Hospital Length of Stay, Log-2 transformed, days & 5.386 & 4.287 & 6.485 & $<0.001$ \\
\hline Hospital Length of Stay > 7 days (Baseline: 7 days or less) & 7.404 & 5.060 & 9.749 & $<0.001$ \\
\hline Total number of $\mathrm{CT}$ scans & 7.898 & 7.595 & 8.201 & $<0.001$ \\
\hline More than 1 CT scan (Baseline: 0 or 1 CT scan) & 16.613 & 14.918 & 18.308 & $<0.001$ \\
\hline RAPID Score & 0.119 & -0.546 & 0.785 & 0.725 \\
\hline RAPID Score = 3-4 (Baseline: 0-2) & -1.312 & -3.507 & 0.882 & 0.241 \\
\hline RAPID Score = 5-7 (Baseline: 0-2) & 1.072 & -1.993 & 4.138 & 0.492 \\
\hline Age at Admission, years & -0.061 & -0.120 & -0.003 & 0.041 \\
\hline Chest tube duration, days & 0.032 & 0.001 & 0.064 & 0.045 \\
\hline Hospital (Baseline: Community) & 3.059 & -0.054 & 6.173 & 0.054 \\
\hline Evidence of loculation on first chest $C T$, yes $(\operatorname{Ref}=\mathrm{No})$ & -2.552 & -4.928 & -0.175 & 0.035 \\
\hline Abscess or necrotizing pneumonia, yes $(\operatorname{Ref}=\mathrm{No})$ & 2.935 & 0.173 & 5.696 & 0.037 \\
\hline CT guided tube thoracostomy, yes $(\operatorname{Ref}=\mathrm{No})$ & 7.431 & 5.087 & 9.775 & $<0.001$ \\
\hline Fluoroscopic guided tube thoracostomy, yes $(\operatorname{Ref}=\mathrm{No})$ & 8.691 & 5.287 & 12.095 & $<0.001$ \\
\hline \multicolumn{5}{|l|}{ Multivariate linear regression analysis: model 1} \\
\hline Surgical Treatment (Baseline: Medical) & -5.314 & -7.289 & -3.338 & $<0.001$ \\
\hline Crossover Treatment (Baseline: Medical) & 2.159 & -0.714 & 5.032 & 0.140 \\
\hline Hospital Length of Stay, Log-2 transformed, days & 3.742 & 2.696 & 4.788 & $<0.001$ \\
\hline Age at Admission, years & -0.055 & -0.105 & -0.005 & 0.032 \\
\hline Evidence of loculation on first chest $C T$, yes $(\operatorname{Ref}=\mathrm{No})$ & -1.490 & -3.527 & 0.548 & 0.152 \\
\hline Abscess or necrotizing pneumonia, yes $(\operatorname{Ref}=\mathrm{No})$ & -0.326 & -2.751 & 2.099 & 0.792 \\
\hline CT guided tube thoracostomy, yes $(\operatorname{Ref}=\mathrm{No})$ & 5.371 & 3.260 & 7.482 & $<0.001$ \\
\hline Fluoroscopic guided tube thoracostomy, yes $(\operatorname{Ref}=\mathrm{No})$ & 6.388 & 3.334 & 9.442 & $<0.001$ \\
\hline \multicolumn{5}{|l|}{ Multivariate linear regression analysis: model 2} \\
\hline Surgical Treatment (Baseline: Medical) & -7.155 & -9.086 & -5.224 & $<0.001$ \\
\hline Crossover Treatment (Baseline: Medical) & 0.974 & -1.952 & 3.900 & 0.513 \\
\hline Hospital Length of Stay, Log-2 transformed, days & 4.162 & 3.094 & 5.230 & $<0.001$ \\
\hline Age at Admission, years & -0.051 & -0.102 & 0.000 & 0.052 \\
\hline Evidence of loculation on first chest $C T$, yes $(\operatorname{Ref}=\mathrm{No})$ & -1.516 & -3.615 & 0.583 & 0.156 \\
\hline Abscess or necrotizing pneumonia, yes $(\operatorname{Ref}=\mathrm{No})$ & 0.219 & -2.273 & 2.711 & 0.863 \\
\hline
\end{tabular}

The following variables were significantly associated with a lower CED; undergoing initial surgical management of CPSI, increased age, and evidence of loculation on initial CT scan. Multivariate linear regression analysis (Model 1-removal of total CT scans and more than 1 CT scan performed variables) identified that increased hospital length of stay and the use of CT or fluoroscopic guidance for chest tube placement is associated with a higher
CED, whereas initial surgical management of CPSI and increased age is associated with a lower CED. When additional multivariate linear regression analysis (Model 2 ) is run with removal of all ionizing radiation variables (total CT scans, more than 1 CT scan performed, use of CT or fluoroscopic guidance for chest tube placement) increased hospital length of stay remains associated with a higher CED, whereas initial surgical management of CPSI remains associated with a lower CED. 


\section{Discussion}

We identified the number of radiographic studies and the overall CED in patients hospitalized for CPSI was high. Recent publications suggest annual environmental radiation dose exposure within the United States around 3 $\mathrm{mSv}[3,17]$. Median CED within our population was $>5$ times that amount, with more than $97 \%$ of our population receiving $5 \mathrm{mSv}$ or more during their admission.

Ionizing radiation utilizing modern radiology techniques has tremendous benefits, likely revolutionizing medical care in the present time. However, this improvement and access to technology is likely not without side effects or risks. There remains debate regarding the true "risk" of low level/dose radiation exposures, however the literature has some data to draw on, including data available from Japanese atomic bomb survivors. Individuals receiving dose ranges of $5-100 \mathrm{mSv}$ (mean exposure of $29 \mathrm{mSv}$ ), had significant increases in solid-cancer incidence when compared to those exposed $<5 \mathrm{mSv}$ [18], suggesting some increased carcinoma risk, even with these lower dose exposures. Despite this the controversy remains as to the true "risk" associated with low dose exposure and what constitutes a low dose exposure. However, most governing bodies accept that radiation induced risks/effects are real (including doses $<100 \mathrm{mSv}$ ), likely have a linear relationship to exposure, and there is likely no completely "safe" dose [19]. Recommendations from both the National Council on Radiation Protection and Measurements and the International Commission on Radiation Protection suggest radiation dosing be kept as low as reasonably achievable [20], the so-named ALARA principle.

Prior studies report varying radiation exposures during hospitalizations, but most have been focused on ICU stays and/or trauma evaluations. Lutterman et al. identified 200 inpatients receiving a mean dose of 14.8 $\mathrm{mSV}$, with significantly higher doses in those spending time in the ICU [11]. Moloney et al. identified $421 \mathrm{ICU}$ patients receiving a median CED of $1.5 \mathrm{mSV}$, with the highest median CED found in trauma patients $(7.7 \mathrm{mSv})$ [10]. Krishnan et al. identified 4155 medical ICU patients reporting a median CED of $0.72 \mathrm{mSV}$, of note, within their population, three percent did receive $>50 \mathrm{mSv}$ [9]. Kim et al. identified critically ill trauma patients requiring prolong ICU stays (> 30 days), reporting a mean CED of $106 \mathrm{mSv}$ per patient [4].

We similarly report on significant radiation exposure in a population of inpatients being managed for CPSI. The majority of radiation exposure (>90\% of CED) during CPSI management appears related to diagnostic CT scan imaging (CT chest or CT angiogram of the chest).

The ability to ascertain why imaging was ordered within a retrospective multi-institutional review remains a significant limitation of our study. Presumably all ordered imaging was "necessary", however reviewing charts retrospectively does not allow for one to reliably identify the reason for ordering imaging. Reviewing progress notes as well as orders within an electronic medical record can provide documentation, however the interpretation of such information in a retrospective manner is likely unreliable and potentially misleading. Advanced imaging modalities have likely led to improved detection and the ability to successfully manage certain conditions, perhaps with CPSI being one of them. We have identified that a majority of patients being managed for CPSI are receiving large amounts of ionizing radiation, including frequent CT imaging. Prior studies, such as the MIST 2 Trial [21], identified chest $\mathrm{x}$-ray imaging as a potential endpoint for determining improvement in pleural disease. Within their trial there was no dictum for utilizing chest $\mathrm{x}$-ray vs CT for CPSI evaluation, rather chest $\mathrm{x}$-ray was simply their primary research endpoint as an objective measure. Interventions such as modifying practice habits to limit chest imaging frequency, utilizing low dose CT imaging, or use of non-ionizing chest imaging (pleural ultrasound) may help minimize radiation exposure to patients, however the clinical impact of such a change is currently unknown. Proposals such as these would be reasonable as we would offer comparison to other situations in which radiographic imaging was considered standard, but over time have fallen to the wayside after evidence of potential harm and continued safety without imaging has been demonstrated. Examples include the use of fluoroscopy during bronchoscopy with transbronchial biopsy and daily chest $\mathrm{x}$-ray use for ventilated patients in the ICU. Fluoroscopy has been and is likely still taught as the standard at many institutions, however, multiple studies have suggested it is unnecessary $[22,23]$ - and not using it can lead to decreased radiation exposure to both patients and staff. Daily chest x-ray use for ventilated ICU patients or after recent pulmonary resection had also been considered a "gold standard" necessary test. However, more recent data suggests that this notion may be untrue and imaging without clinical concern may be unnecessary $[24,25]$.

Non-ionizing radiation techniques, such as pleural ultrasound have been helpful in the management of pleural disease [26, 27], however we are unaware of any data suggesting its utility in the more longitudinal management of inpatients with CPSI and how it may fare against other modalities such as chest x-ray or chest CT. An additional limitation of our study was the inability to capture the number of ultrasound guided procedures during CPSI management. Anecdotally, we are aware that some ultrasound guided tube thoracostomy is performed, it remains unclear as to the availability and/or expertise 
of pleural ultrasound within a larger healthcare system network [27, 28]. It also remains unclear as to how and/ or if longitudinal pleural ultrasound is utilized. Numerous variables appear associated with CED on univariate linear regression analysis. However, due to concerns related to collinearity and variables of ionizing radiation exposure we decided to remove these variables during multivariate analysis. In model $1-$ the use of CT and fluoroscopic guidance are associated with increased CED, however we again suggest caution in the interpretation of this related to previous concerns of collinearity. After removal of all ionizing radiation variables it appears that hospital length of stay is the most predictive of increased CED (in both models), consistent with the likely need for longer stay suggesting more complicated disease process and therefore likely additional imaging to manage more complicated disease. Initial surgical intervention and increased age remain associated with decreased CED. It remains unclear as to the association of increased age and decreased CED. We identified additional variables such as RAPID score, Age, and BMI impacted treatment modalities (data not shown) but found no significant associations. We can suggest that perhaps increased age is associated with less aggressive treatment, however as just noted, we were unable to provide any evidence to support such. In light of the retrospective nature of this study it does remain difficult to make any additional associations.

This study has several limitations. It is a retrospective review and all of the inherent bias and shortcomings associated with such studies. One shortcoming in retrospective studies is often incomplete medical records and inability to determine clinical decision making. While we were unable to perform the latter, regarding the former we feel fairly confident that the use of a system-wide, unified, electronic medical record allows us to accurately obtain our specified datapoints with tremendous accuracy. We suspect that our radiation exposure values likely vary slightly from institution and from patient, however our CED values are calculated from published values previously utilized [15] potentially making them fairly generalizable.

In conclusion, we identified a large number of radiographic studies being performed during the inpatient management of CPSI, with a resultant high CED. The median CED within our population was greater than 5 times the normal yearly radiation exposure, and $74 \%$ of the population received more than $10 \mathrm{mSv}$ during their stay. While we wholeheartedly agree that modern radiology techniques have tremendous benefits and have revolutionized medical care, they are likely not without side effects or risks. We would suggest ongoing evaluation of CED during admission for CPSI, including consideration of expanding to other disease processes that may place patients and healthcare staff at excessive risk of radiation exposure. One potential alternative strategy to high radiation exposure imaging (such as standard CT scans) could include the use of widespread pleural ultrasound for both initial evaluation and longitudinal follow-up. Other potential strategies could include the use of low dose CT scan imaging for subsequent follow-up imaging, and/or protocol driven imaging attempting to limit overall radiation exposure during hospital admission. Additional study is warranted to identify the frequency and type of imaging needed in the management of complex pleural space infections as an attempt to keep ionizing radiation exposure as low as reasonably possible.

\section{Abbreviations}

CPSI: Complex pleural space infections; CED: Cumulative effective dose; IPFT: Intrapleural fibrinolytic therapy; mSv: Millisievert; Sv: Sievert; Gy: Gray; IQR: Interquartile range; CT: Computed tomography; DLP: Dose length product; DAP: Dose area product; LOS: Length of stay; ALARA: As low as reasonably achievable; BMI: Body mass index; ICU: Intensive care unit.

\section{Acknowledgements \\ None.}

\section{Authors' contributions}

CRG is the guarantor of the paper, taking responsibility for the integrity of the work as a whole, from inception to published article. CRG, ASJ, CLW, LCH, SC, AJB, EV, JAG contributed in conception and design, provision of study patients, collection of data, data analysis and interpretation, manuscript writing, and final approval of manuscript.

\section{Funding}

Support for this project was provided from The Center for Lung Research in honor of Wayne Gittinger.

\section{Availability of data materials}

The datasets analyzed during the current study can be made available by the corresponding author on reasonable request.

\section{Declarations}

Ethics and consent to participate

This study was approved by the Swedish Medical Center IRB under Study\# Study\# 2018000200. All study methods were performed in accordance with relevant guidelines, practices, and regulations related to performing human subject research at Swedish Medical Center.

\section{Consent to publication}

CRG, ASJ, CLW, LCH, SC, AJB, EV, JAG have all reviewed the final manuscript and agree to consent to publication.

\section{Competing interest}

The authors declare that they have no competing interests.

\section{Author details}

${ }^{1}$ Thoracic Surgery and Interventional Pulmonology, Swedish Cancer Institute, 1101 Madison St, Suite 900, Seattle, WA 98104, USA. Department of Surgery, Medstar Georgetown University Hospital, Washington, DC, USA. ${ }^{3}$ Medical Data Research Center, Providence St. Joseph Health, Portland, OR, USA.

Received: 15 January 2021 Accepted: 30 March 2021

Published online: 23 April 2021 


\section{References}

1. Davies CWH. BTS guidelines for the management of pleural infection. Thorax. 2003;58:18ii-28.

2. Shen KR, Bribriesco A, Crabtree T, et al. The American Association for Thoracic Surgery consensus guidelines for the management of empyema. J Thorac Cardiovasc Surg. 2017;153:e129-46.

3. Brenner DJ, Doll R, Goodhead DT, et al. Cancer risks attributable to low doses of ionizing radiation: assessing what we really know. Proc Natl Acad Sci USA. 2003;100:13761-6.

4. Kim PK, Gracias VH, Maidment ADA, O'Shea M, Reilly PM, Schwab CW. Cumulative radiation dose caused by radiologic studies in critically ill trauma patients. J Trauma Injury Infect Crit Care. 2004;57:510-4.

5. Howard A, West R, Iball G, Pantelo M, Pandit H, Giannoudis PV. An Estimation of lifetime fatal carcinogenesis risk attributable to radiation exposure in the first year following polytrauma: a major trauma center's experience over 10 years. J Bone Joint Surg. 2019;101:1375-80.

6. Brinkman AS, Gill KG, Leys CM, Gosain A. Computed tomography-related radiation exposure in children transferred to a Level I pediatric trauma center. J Trauma Acute Care Surg. 2015;78:1134-7.

7. Smith-Bindman R, Lipson J, Marcus R, et al. Radiation dose associated with common computed tomography examinations and the associated lifetime attributable risk of cancer. Arch Intern Med. 2009;169:2078-86.

8. Miglioretti DL, Johnson E, Williams A, et al. The use of computed tomography in pediatrics and the associated radiation exposure and estimated cancer risk. JAMA Pediatr. 2013;167:700-7.

9. Krishnan S, Moghekar A, Duggal A, et al. Radiation exposure in the medical ICU: predictors and characteristics. Chest. 2018;153:1160-8.

10. Moloney F, Fama D, Twomey M, et al. Cumulative radiation exposure from diagnostic imaging in intensive care unit patients. World J Radiol. 2016:8:419-27.

11. Lutterman A, Moreno CC, Mittal PK, Kang J, Applegate KE. Cumulative radiation exposure estimates of hospitalized patients from radiological imaging. Am Coll Radiol. 2014;11:169-75.

12. Rahman NM, Kahan BC, Miller RF, Gleeson FV, Nunn AJ, Maskell NA. A clinical score (RAPID) to identify those at risk for poor outcome at presentation in patients with pleural infection. Chest. 2014;145:848-55.

13. Corcoran JP, Psallidas I, Gerry S, et al. Prospective validation of the RAPID clinical risk prediction score in adult patients with pleural infection: the PILOT study. Eur Respir J. 2020;56:2000130.

14. Allisy-Roberts PJ. Radiation quantities and units-understanding the sievert. J Radiol Prot. 2005;25:97-100.
15. Mettler FA, Huda W, Yoshizumi TT, Mahesh M. Effective doses in radiology and diagnostic nuclear medicine: a catalog. Radiology. 2008;248:254-63.

16. Radiation doses in interventional fluoroscopy. 2019. https://www.iaea. org/resources/rpop/health-professionals/interventional-procedures/radia tion-doses-in-interventional-fluoroscopy\#2. Accessed 9 Dec 2019

17. Committee to Assess Health Risks from Exposure to Low Levels of Ionizing Radiation NRC. Health Risks from Exposure to Low Levels of lonizing Radiation 2006

18. Pierce DA, Preston DL. Radiation-related cancer risks at low doses among atomic bomb survivors. Radiat Res. 2000;154:178-86.

19. Hall EJ, Brenner DJ. Cancer risks from diagnostic radiology. Br J Radiol. 2008;81:362-78.

20. Dainiak N. Radiation dose and stochastic risk from exposure to medical imaging. Chest. 2013;144:1431-3.

21. Rahman NM, Maskell NA, West A, et al. Intrapleural use of tissue plasminogen activator and DNase in pleural infection. N Engl J Med. 2011;365:518-26.

22. Anders GT. Transbronchial biopsy without fluoroscopy. A seven-year perspective. CHEST J. 1988;94:557.

23. De Fonoyl O, Capron F, Lebeau B, Rochemaure J. Transbronchial biopsy without fluoroscopy: a five year experience in outpatients. Thorax. 1989:44:956-9.

24. Graat ME, Choi G, Wolthuis EK, et al. The clinical value of daily routine chest radiographs in a mixed medical-surgical intensive care unit is low. Crit Care (Lond, Engl). 2006:10:R11.

25. Cerfolio RJ, Bryant AS. Daily chest roentgenograms are unnecessary in nonhypoxic patients who have undergone pulmonary resection by thoracotomy. Ann Thorac Surg. 2011;92:440-3

26. Millington SJ, Koenig S. Better with ultrasound: pleural procedures in critically ill patients. Chest. 2018;153:224-32.

27. HavelockT, Teoh R, Laws D, Gleeson F. Pleural procedures and thoracic ultrasound: British Thoracic Society Pleural Disease Guideline 2010. Thorax. 2010;65(Suppl 2):ii61-76.

28. National Patient Safety Agency. Rapid response report risks of chest drain insertion. In: Division NHSPS. London: National Health Service; 2008:1-10.

\section{Publisher's Note}

Springer Nature remains neutral with regard to jurisdictional claims in published maps and institutional affiliations.
Ready to submit your research? Choose BMC and benefit from:

- fast, convenient online submission

- thorough peer review by experienced researchers in your field

- rapid publication on acceptance

- support for research data, including large and complex data types

- gold Open Access which fosters wider collaboration and increased citations

- maximum visibility for your research: over $100 \mathrm{M}$ website views per year

At $\mathrm{BMC}$, research is always in progress.

Learn more biomedcentral.com/submissions 